\title{
TRANSFORMACIONES A PARTIR DEL ESPACIO DE FORMACION INTEGRAL: "TALLER ABIERTO DE LECTURA, INTERPRETACIÓN Y CREACIÓN EN TORNO A LITERATURAS NO REALISTAS, INSÓLITAS Y FANTÁSTICAS” EN EL HOSPITAL VILARDEBÓ
}

\section{THE TRANSFORMATIONS OBTAINED THROUGH THE COMPREHENSIVE EDUCATION SPACE: "OPEN WORKSHOP FOR READING, INTERPRETATION AND CREATION AROUND NON-REALISTIC, EXTRAORDINARY AND FANTASTIC LITERATURES” AT VILARDEBÓ HOSPITAL}

Hebert Benitez Pezzolano* Estefanía Pagano Artigas**

Resumen: Desde el año 2015, se lleva a cabo el Espacio de Formación Integral del Departamento de Letras de la Facultad de Humanidades y Ciencias de la Educación de la Universidad de la República (Uruguay). El mismo, denominado "Taller abierto de lectura, interpretación y creación en torno a literaturas no realistas, insólitas y fantásticas", se desarrolla en el Hospital Vilardebó. Este revela, en tanto práctica de extensión, diversas transformaciones que merecen ser percibidas, sometidas a reflexión, escritas y compartidas. Es así que en el presente artículo se da a conocer dicha experiencia de extensión universitaria enmarcada en los aportes teóricos que la Universidad de la República ha generado sobre Extensión, su metodología, sus atravesamientos y sus transformaciones.

Palabras clave: EFI; transformaciones; literaturas no realistas.

\begin{abstract}
Since 2015, it is carried out through the "Comprehensive Education Space" of the Department of Literature of the Faculty of Humanities and Education Sciences of University of Republic of Uruguay the activity "Open workshop for reading, interpretation and creation around non-realistic, extraordinary and fantastic literatures". It is developed at Vilardebó Hospital and demonstrates, as an extension practice, various transformations that deserve to be perceived, reflected, written and shared. Thus, this paper presents the experience of this university extension activity which was conducted through the theoretical contributions that University of the Republic has produced about extension, its methodology, its intersections and its transformations.
\end{abstract}

Keywords: Comprehensive education; transformations; non-realistic literatures. 


\section{Introducción}

En el presente artículo intentaremos compartir una experiencia de extensión universitaria del Departamento de Literaturas Uruguaya y Latinoamericana de la Facultad de Humanidades y Ciencias de la Educación de la Universidad de la República (FHUCE-UdelaR) de Uruguay bajo el dispositivo de un Espacio de Formación Integral (EFI). Dichos dispositivos, que la UdelaR viene implementando durante los últimos diez años, buscan hacer operativas las prácticas integrales universitarias a partir de favorecer el desarrollo de sus principales elementos: integración de las funciones universitarias (enseñanza, investigación y extensión); abordajes interdisciplinarios para el aporte a la solución de problemas sociales; diálogo de saberes como base para una intencionalidad transformadora; enfoque territorial e intersectorial de las intervenciones (UNIVERSIDAD DE LA REPÚBLICA, 2010).

Compartir una experiencia no sólo implica dar a conocer una experiencia en el marco de la extensión universitaria sino animarse a llevar a la escritura pensamientos y reflexiones que toda experiencia extensionista promueve. Dichas reflexiones convocan, por un lado, a crear nuevos conocimientos particulares de la propia experiencia pero que por otro lado convocan a profundizar el proceso que desde el 2006 ha tenido la Universidad de la República: la integración de la extensión con la investigación y la importancia de la primera para la formación universitaria en la vinculación con la sociedad.

\section{Se trata de Extensión}

La extensión universitaria, según la Resolución del Consejo Directivo Central de la Universidad de la República es:

-Proceso educativo transformador donde no hay roles estereotipados de educador y educando, donde todos pueden aprender y enseñar. (...)

-Proceso que contribuye a la producción de conocimiento nuevo, que vincula críticamente el saber académico con el saber popular.

-Proceso que tiende a promover formas asociativas y grupales que aporten a superar problemáticas significativas a nivel social.

- Es una función que permite orientar líneas de investigación y planes de enseñanza, generando compromiso universitario con la sociedad y con la resolución de sus problemas

-En su dimensión pedagógica, constituye una metodología de aprendizaje integral y humanizadora (UNIVERSIDAD DE LA REPÚBLICA, 2010, 15,16).
Se trata entonces de un proceso, una función, que desde el conjunto de sus herramientas metodológicas busca promover la Integralidad. Integralidad de extensión, investigación y formación; de los actores sociales y universitarios; del "adentro" de la Universidad y del "afuera" de la misma (TOMMASINO; RODRÍGUEZ, 2011). Pensamos a la extensión como "una razón continua, crítica e integrada en el seno de las funciones universitarias y de los protagonistas académicos y de la comunidad" (BENÍTEZ, 2016: 32). Así lo exponen Cano y Romano (2015):

\begin{abstract}
No son demasiados los investigadores universitarios que desde diferentes ramas de conocimiento aportan hoy al pensamiento crítico con vocación superadora de los límites del neodesarrollismo capitalista hegemónico. Pero tampoco son pocos. Están por toda la Universidad, en diferentes facultades y áreas de conocimiento. Varios de ellos están en Extensión. Investigan sobre las consecuencias sociales, ecológicas y productivas del modelo de agronegocio dominante; sobre las condiciones de trabajo de los trabajadores de la industria pesquera; sobre las consecuencias sociales y urbanas de los barrios privados; sobre las memorias barriales respecto a las luchas sociales pasadas; sobre las políticas económicas alternativas necesarias para acrecentar el presupuesto educativo; sobre las políticas energéticas y la actual distribución desigual de sus costos entre la población; sobre las implicaciones del proyecto agro-industrial del norte del país y el lugar que en ellos le cabe a los trabajadores; sobre las cooperativas de vivienda; sobre las condiciones de vida y trabajo de los clasificadores de residuos urbanos sólidos; sobre la comunicación en las organizaciones sindicales; sobre los desafíos de la autogestión en las organizaciones sociales; sobre agro-ecología, cooperativismo, feminismo y sindicalismo. (CANO, ROMANO, 2015).
\end{abstract}

\section{Espacio de Formación Integral}

El Espacio de Formación Integral (de ahora en más EFI) "Taller abierto de lectura, interpretación y creación en torno a literaturas no realistas, insólitas y fantásticas" en el Hospital Vilardebó es una práctica de extensión universitaria que se desarrolla en los semestres pares desde el año 2015. Está coordinado desde el Departamento de Literaturas Uruguaya y Latinoamericana de la Facultad de Humanidades y Ciencias de la Educación de la Universidad de la República y se enmarca en el proyecto de investigación "Raros y Fantásticos en la Literatura Uruguaya. Historia, crítica y teoría (1963-2004)", así como también con los seminarios de literatura uruguaya de dicho Departamento. Por Espacio de Formación Integral entendemos una serie de

dispositivos flexibles que se conforman a partir de múltiples experiencias educativas en diálogo con la sociedad, prácticas, cursos, talleres, pasantías, proyectos de extensión y/o investigación, asumiendo 
diferentes formas de reconocimiento curricular según las características de cada servicio universitario (TOMMASINO, RODRÍGUEZ, 2011, 20).

En el EFI participa el equipo docente que coordina el mismo (compuesto por una Profesora de Literatura y una Licenciada en Psicología), las pacientes del hospital del área de internación y los estudiantes universitarios. El EFI puede ser cursado por estudiantes de todas las Licenciaturas (Filosofía, Letras, Historia, Educación, Ciencias Antropológicas, Lingüística, Biología Humana, Turismo y Binacional en Turismo) y Tecnicaturas (Corrección de Estilo, Universitaria en Museología, Tecnólogo en Interpretación y traducción LSU- Español, Universitaria en Bienes Culturales, Universitaria en Dramaturgia) de la facultad de Humanidades y Ciencias de la Educación así como también por estudiantes de la Licenciatura de Psicología de la Facultad de Psicología de la Universidad de la República. Este último apoya la siguiente tesis elaborada por Tommasino y Rodríguez (2011):

\begin{abstract}
La realidad es indisciplinada. La intervención para su transformación debe ser necesariamente interdisciplinaria (..) El diálogo interdisciplinar, originariamente académico, debe incluir, ser criticado y criticar el saber popular, conformando una red de saberes, una ecología de saberes que contribuye a la transformación participativa de la realidad. (TOMMASINO, RODRÍGUEZ, 2011: 38)
\end{abstract}

El objetivo general de este EFI es propiciar una actividad de debate en torno a textos no realistas en la que las integrantes del mismo experimenten creaciones de imaginación artística, así como también de cuestionamientos de cierta idea de realidad y/o de ficción (BENÍTEZ, 2017). En cuanto a la formación, busca acercarse a lo interdisciplinario con la psicología. En lo académico, intenta por un lado promover la familiarización de los estudiantes así como también de las usuarias con las literaturas no realistas. Por otro lado, reconocer y valorar el desafío de otras interpretaciones, en este caso, de las mujeres que por determinada circunstancia de la vida se encuentran internadas en el Hospital Vilardebó, interpretaciones que se hallan fuera de la academia. Por último busca también contribuir a la comunidad, es decir, apuntar a cierto efecto terapéutico.

En concreto, el EFI consiste en dos actividades. Los talleres de lectura en el Hospital Vilardebó en los que se leen textos literarios narrativos uruguayos no realistas, insólitos y fantásticos realizándose un permanente debate, una continua comprensión y posterior interpretación. Al igual que Romano,

No estamos ahí para trabajar sobre la historia ni los dichos de los pacientes ni ninguna otra cosa más que realizar la tarea que nos convoca (...) La propuesta es que puedan disfrutar, hacer, jugar en la acción, en un intento de acotar el goce al cual su estructura los somete; que se produzca algo del orden del placer. (ROMANO, 2007, 99,100)

Los encuentros teóricos en la Facultad comenzaron siendo una vez por mes y actualmente funcionan quincenalmente. En las mismas se abre el espacio a la problematización de las experiencias vividas en el hospital, no sólo en tanto estudiantes universitarios sino también en tanto integrantes de una sociedad, con una historia de vida y ciertas perspectivas de análisis. Asimismo se discuten textos teóricos que giran alrededor de las temáticas de los derechos humanos, de las psicosis y de las literaturas no realistas.

Taller y encuentro son las distintas metodologías que lleva a cabo el EFI. En cuanto al taller, éste se entiende como

\begin{abstract}
dispositivo de trabajo con grupos, que es limitado en el tiempo y se realiza con determinados objetivos particulares, permitiendo la activación de un proceso pedagógico sustentado en la integración de teoría y práctica, el protagonismo de los participantes, el diálogo de saberes, y la producción colectiva de aprendizajes, operando una transformación en las personas participantes y en la situación de partida (CANO, 2012, 30).
\end{abstract}

En sintonía con las observaciones generales de Cano, el taller en el hospital es un dispositivo realizado en grupos, que es limitado en el tiempo ya que comienza en agosto y finaliza en diciembre, realizándose con los objetivos anteriores escritos, permitiendo la activación de un proceso pedagógico horizontal entre el equipo docente, los estudiantes universitarios y las usuarias del hospital integrando de manera constante la teoría y la práctica, promoviendo el protagonismo de los participantes, en este caso las pacientes del hospital que asisten al taller y devienen usuarias talleristas, la producción de un conocimiento colectivo nuevo creado a partir de las reflexiones, de las interpretaciones y del debate mediante la lectura del texto operando una transformación en la experiencia íntegra. En cuanto al encuentro teórico, vale destacar que se continúa buscando un nombre para dicho espacio. Al comienzo eran reuniones, luego pasaron a ser sesiones y actualmente se les llama encuentros teóricos. Encuentro en tanto acto de reunión en el que se ponen en juego nuestras historias de vida a la hora de pensar y problematizar aquellos momentos o sucesos que nos llamaron la atención del taller, así como también al momento de complejizar ciertos textos teóricos teniendo como horizonte la práctica. Al decir Foucault: "Ninguna teoría puede desarrollarse sin encontrar una especie de muro, y se precisa la práctica para agujerearlo" (FOUCAULT, 2003: 84). 


\section{La locura y la literatura no realista}

El taller de lectura e interpretación se desarrolla en el Hospital Vilardebó, que es el único hospital psiquiátrico público de referencia de pacientes agudos con que cuenta el Uruguay. De ahí que reciba personas de todo país, lo que lo convierte en un hospital con una población variada en su procedencia geográfica y socio-económica y cultural. El edificio cuenta con una Policlínica de Salud Mental, un Centro Diurno, la Emergencia y el área de internación dividido en dos grandes sectores: uno destinado para las mujeres, sector femenino y otro para los hombres, sector masculino. Si el Hospital Vilardebó es un hospital psiquiátrico, el taller se realiza en una institución manicomial con todas las características que éste presenta: lógica de encierro, aislamiento, exclusión, pobreza, destrato, olvido, abandono y deshumanización. Goffman (1961), realiza un estudio en relación a las Instituciones de encierro como los manicomios y las cárceles, entre otros, y como el encierro propiamente dicho afecta el ser humano. Según este autor, este tipo de instituciones están condenadas al fracaso, ya que en lugar de promover la recuperación/rehabilitación, lo que logran al final es promover la estigmatización de las personas y terminan cronificando las patologías. En estas situaciones el hospital llamado de agudos pasa a convertirse en un hospital de crónicos.

El taller se realiza en el sector femenino de internación, en el Espacio Psicosocial. El mismo es un lugar dentro del área de internación destinado a distintas actividades de rehabilitación con pase libre, es decir, sin necesitar del pase del psiquiatra tratante. Para el hospital, entonces, el taller es una actividad de rehabilitación. Con respecto al concepto de rehabilitación, Techera, Apud Borges (2009) plantean que existen dos maneras de entender la rehabilitación. Desde una concepción más tradicional tenemos al enfoque normativo, el cual entiende a ésta como un restablecimiento de la normalidad, una normalidad preestablecida por la sociedad. Desde una concepción más actual se encuentra el enfoque paradigmático, el cual concibe a la rehabilitación como un proceso que facilita a las personas con alguna limitación, a reorganizar de una manera comprensiva la autonomía de sus funciones en la comunidad. Según Rodríguez y Sobrino (1999) la rehabilitación es un proceso que prepara al sujeto a la reinserción en la sociedad, mientras que se van practicando habilidades y potencialidades para su autonomía. Para Van Weenghel y Kroon (2004) la rehabilitación comprende
El Espacio Psicosocial es un lugar físico de ostensible abandono. El mismo consiste en un pasillo largo y angosto, al costado de una sala, con una monstruosa humedad que gotea cada vez que llueve. Las temperaturas parecen que se exacerban, haciendo muchísimo calor en verano (lo que generó que en muchas oportunidades la actividad se haya realizado en el patio) y muchísimo frío en invierno. Asimismo escasean los materiales (hojas, lápices, marcadores) y faltan sillas. No es por casualidad que el espacio destinado para la rehabilitación en las mujeres internadas sea un lugar muy abandonado. Revela el lugar que tiene la rehabilitación en el manicomio y el lugar de rehabilitación en la mujer. Lugares que no son lugares (no olvidemos que el espacio es un pasillo); lugares olvidados, abandonados, no priorizados por la lógica manicomial.

Es en el llamado Espacio Psicosocial que la literatura no realista se presenta y el debate e interpretación aflora.

Para señalarlo sintéticamente, las literaturas no realistas, insólitas y fantásticas son aquellas que entablan alguna clase de conflicto entre lo que comúnmente se asocia con lo real y lo irreal, así como con sus códigos más extendidos. En los estudios literarios cobra cierta centralidad la oposición categorial realista/fantástico, aunque una limitación en esos términos resultaría reduccionista, hecho que en la práctica del taller y en las sesiones teóricas ha sido puesto de relieve. Este último se crea a partir de su frontera con lo real, supera esta misma por su contradicción con lo real; más que la supera, la contradice y la transgrede (CAMPRA, 2008). Estas literaturas representan un conflicto que rompe con cierta tradición literaria realista, en la que de uno $\mathrm{u}$ otro se revela un orden medianamente estable. Estas literaturas son y no son realistas al mismo tiempo, desafiando así las categorías modernas que delimitan y se diferencian de lo otro, de lo que no es. Según Pagano, lo fantástico

\footnotetext{
comienza en el autor, ya sea consciente o inconsciente de lo que está produciendo y de la manera en que produce, que se desprende luego al texto pasando a ser este una producción ficcional autónoma que vive la convivencia de lo que la historia, el mundo posible creado considera como posible / imposible, normal / anormal, animado / inanimado, concreto / abstracto; como un conflicto que tiende en general a no resolverse y a subyacer (...). El propio texto guarda en sí mismo una resonancia para entender y aceptar que otros mundos, otras realidades diferentes, son posibles. (PAGANO, 2015: 3)
}

la revaloración de pacientes como ciudadanos (misión) al entrenamiento de habilidades y apoyo (método), a la voluntad de centrarse en las necesidades y los objetivos de los pacientes (actitud) y a la transición de una atención basada en el hospital a un sistema de atención comunitaria (organización de ciudadanos)" (VAN WEENHEL, KROON, 2004, 73).
Sin dejar de tener en cuenta que nos encontramos ante mundos posibles de la ficción literaria, sean estos realistas, hiperrealistas, fantásticos o de una extrañeza que resiste a la relativa estabilidad 
que imprimen estas categorías, digamos que aquellos textos que se deja nombrar como "fantásticos", incluso de un modo muy general, contienen cosas y entablan procesos que no se permiten en el orden estabilizado como real, o natural, o empírico. Entre otras definiciones tentativas puede pensarse que lo fantástico se identifica con

\begin{abstract}
aquella producción ficcional en la que se establece un problema o conflicto originado entre lo que para ese texto y las ideologías que lo habilitan son lo posible y lo imposible en tanto que acontecimientos, es decir, en tanto que factualidades de presentación excluyente en el interior de ese mundo ficcional y de representación también excluyente en la proyección sobre mundos a los que históricamente atribuimos estatutos de "realidad" (BENITEZ, 2014: 14).
\end{abstract}

Un conjunto significativo de literaturas no realistas suele movilizar la sublevación de lo establecido a través de la convivencia con lo imposible. De ahí, en esos casos, su carácter subversivo; carácter que en muchas ocasiones se percibe a través del silencio de cierta explicación acerca de lo que ocurrió. Es esa subversión dada por la convivencia con lo imposible, por ese silencio que se impone y que no da lugar a la explicación, es esa indefinición lo que genera distintos cuerpos de lo insólito, entre los cuales las manifestaciones de lo fantástico. Según Campra, lo fantástico se manifiesta mediante una isotopía de la transgresión: "la distorsión en el nivel sintáctico, o un uso particular de un elemento del nivel verbal constituye (...) el índice de lo fantástico" (CAMPRA, 2008: 194).

A través de la práctica de extensión que venimos presentando; a través del EFI recientemente descripto es que la locura y las literaturas no realistas se encuentran, se entrecruzan, se atraviesan y confluyen a través de la extensión de una manera por demás particular: dándole espacio a la locura se observa una recepción de dichas literaturas diferente a la que se conoce. La locura revela un agujero teórico en las literaturas no realistas, el cual transforma realizando en las prácticas de su propia recepción nuevas inquietudes para la teoría.

\section{Algunas transformaciones}

El concepto de transformación implica un antes, un durante y un después. Implica que algo devenga otra cosa distinta debido a cierto proceso. Transformar es transmutar. Más arriba mencionamos que la extensión, de acuerdo a la resolución del Consejo Directivo Central de la Universidad de la República (2010) y que el taller, de acuerdo a la concepción de Cano (2012), son procesos que generan ciertas transformaciones.

Varias son las transformaciones que se observan en esta práctica de extensión. En primer lugar, las mujeres que asisten al taller no son consideradas como pacientes que presentan cierto diagnóstico psiquiátrico sino usuarias que asisten a un taller de lectura que tienen algo para decir y que sus palabras son tan importantes como las de cualquier otra persona. La palabra de "la loca" en el taller de lectura tiene su lugar. Al decir de Hounie: "Que una palabra tenga lugar significa que se le ha permitido ser en su condición de inesperada, reveladora, intempestiva y potente para construir espacios en los que poder habitar" (HOUNIE, 2013: 120). Que el taller de lectura sea un espacio de escucha, de auténtica escucha en donde la mujer no es paciente sino usuaria, condice con la perspectiva que tienen los psicólogos que trabajan en el Espacio Psicosocial:

En el pequeño espacio logramos colocar materiales para
leer, recortar, pegar, escribir, hay música, la escucha
está pronta y las ganas de compartir también. De eso se
trata, de estar dispuesto a "recibir la psicosis" (término
que, si no me equivoco, se lo escuché en alguna
conferencia a Marcelo Percia (PERDOMO, 2013: 57).

En esa escucha es que las usuarias se transforman en lectoras críticas evidenciando una otra recepción de estas literaturas no realistas. Sus interpretaciones revelan a las literaturas no realistas como otra forma de realismo. En sus análisis, si bien reconocen el conocido límite entre realidad y ficción, el mismo parece no importar: puesto que el eje se ubica en que lo que sucede en los textos, es posible de ser real. Se animan a contestar preguntas y a discutir las dudas del texto, desafían la crítica literaria que ubica lo fantástico en la frontera de lo real y lo irreal, para conceptualizarlo en términos de realismo: "Tiene que ser real", se suele escuchar en los debates de los textos.

El taller también permite cierto proceso de alfabetización en las usuarias con poco nivel cultural. En él se conocen autores uruguayos, se intercambia acerca de la noción y características del género narrativo, se conocen nuevas palabras y expresiones, se incentiva la concentración y la atención (lo que se hace muy difícil en un manicomio). La alfabetización a través del arte, en este caso la literatura, permite, entre otros aspectos, historizarse y poder nombrarse. Así expresan Penino, Perdomo y De León:

El despliegue de lo grupal y lo estético, el arte, nos
parece algo fundamental en el trabajo en Salud Mental
(...) El arte obliga a las personas a un recorrido interior,
a una búsqueda interior de equilibrio, una forma de
enfrentamiento y de procesar y elaborar la realidad.
Genera un clima de afectividad notoria, que en muchas
ocasiones visualizamos que hace "dejar el delirio de
lado". Vemos que las personas al meterse en el campo 
productivo, a realizar "el objeto artístico", compaginan una subjetividad muy diferente. (PENINO, PERDOMO, DE LEÓN, 2013: 223)

Otra transformación que perfectamente puede englobar a las anteriores es la de la pausa o el paréntesis manicomial. En el taller se suspenden las lógicas manicomiales que atraviesan los cuerpos, los pensamientos y sentimientos de las mujeres internadas, promoviéndose la escucha auténtica, la libertad en la expresión, la importancia y respeto en lo que cada una expresa, la horizontalidad e intercambio de saberes. El taller de lectura e interpretación se constituye como una práctica desmanicomializante, en tanto

\begin{abstract}
desmanicomializar implica fundamentalmente desarmar permanentemente la lógica clasificatoria y concebir modelos de convivencia superadores del "canibalismo simbólico". La lógica anti-manicomial debería ser una lucha contra la lógica manicomial allí donde esta encuentre terreno propicio para fijarse, nacer 0 recrearse. (CANO, 2013: 114,115)
\end{abstract}

Por último, (y no menos importante) la transformación no es sólo para las usuarias del taller sino también para el equipo de coordinación y los estudiantes universitarios. Para comenzar el EFI rompe con la lógica tradicional del saber vertical o magistral. En el mismo todos tienen un saber valioso a ser compartido e interactuado con el resto de los actores que lo integran. Cada estudiante, desde la carrera que está estudiando aportan su perspectiva para pensar esta práctica desde la auténtica interdisciplina. Así, por ejemplo, los estudiantes de Psicología se han detenido en pensar el padecer psíquico de las usuarias y cómo oficia la literatura; los de la Licenciatura en Educación en el vínculo entre tallerista/integrante de taller, los de Tecnicatura en Corrección de Estilo y de la Licenciatura de Letras han observado el análisis que hacen las usuarias de los textos leídos, los de la Licenciatura de Filosofía en los conceptos de locura y encierro. Asimismo, los estudiantes en tanto observadores participantes, registran y luego intercambian acerca de lo debatido entre las usuarias a partir de las lecturas de los textos. De esta manera no sólo se observa un pensar la práctica desde la interdisciplina sino un además un diálogo de saberes que mantiene su carácter transformador de una realidad que duele y que se quiere cambiar. La transformación deviene integral no sólo por tratarse de una práctica que busca la integralidad en las funciones universitarias sino porque invade los espacios del estudiante universitario (y del equipo de Coordinación) no solo en tanto estudiantes sino también como personas tal como ellos mismos explicitan en los encuentros teóricos o en los informes que realizan al final de la práctica. Invade en el sentido de generar cuestionamientos en las perspectivas de vida de los mismos. Invade, atraviesa y transgrede.

\section{Consideraciones finales}

El EFI "Taller abierto de lectura, interpretación y creación en torno a literaturas no realistas, insólitas y fantásticas" que se lleva a cabo en el Hospital Vilardebó es una práctica de extensión que acompaña un nuevo proceso de formación en los estudiantes universitarios protagonizado por la integralidad. Asimismo deviene en práctica desmanicomializante, clave para la coyuntura actual de la Salud Mental en el Uruguay (tiempos de una nueva ley de Salud Mental que si bien presenta ciertos avances con respecto a la anterior, de 1934, continúa con la estigmatización del usuario de Salud Mental).

Cada taller y cada encuentro visibiliza nuevos conocimientos y reflexiones, complejidades y desafíos que motivan la continuación del EFI y su problematización constante.

Es tarea de nosotros (escritores/as y lectores/as) contagiar la necesidad de prácticas de extensión que política y culturalmente son fundamentales para el cambio social.

\section{Referencias bibliográficas}

BENÍTEZ PEZZOLANO, Hebert. Raros y fantásticos: perspectivas teóricas. [sic], Montevideo, v. 4, n. 10, p. 1320, abr. 2014.

Una razón extendida. In: FACULTAD DE HUMANIDADES Y CIENCIAS DE LA EDUCACIÓN. 70 años Facultad de Humanidades y Ciencias de la Educación. Montevideo: Universidad de la República, 2016. p. 32-33.

Espacio de Formación Integral: Taller abierto de lectura, interpretación y creación en torno a literaturas no realistas, insólitas y fantásticas. Disponível em: $<$ http://www.fhuce.edu.uy/images/Unidad_de extension/EF I/2017/EFI 2017 Taller abierto de lectura.doc.pdf $>$.

Acesso em: 5 set. 2017.

CAMPRA, Rosalba. Territorios de la ficción. Lo fantástico. Sevilla: Renacimiento, 2008.

CANO, Agustín. La metodología de taller en los Procesos de Educación Popular. Revista Latinoamericana de Metodología de las Ciencias Sociales, La Plata, v. 2, n. 2 , p. 22-51, jul.-dez. 2012.

Algunos desafíos para la desmanicomialización en Uruguay. In: DE LEON, N. (Comp). Abrazos Experiencias y narrativas acerca de la locura y la salud mental. Montevideo: Ediciones Levy, 2013. p. 107-124.

ROMANO, Antonio. La universidad conservadora. A propósito de un artículo de Ferrer y Bolon sobre la extensión universitaria. Disponível em: $<\mathrm{http}$ ///www.fhuce.edu.uy/images/comunicacion/Noticias/2 015/octubre/La_universidad_conservadora.pdf $>$. Acesso em: 3 set. 2017 .

PENINO, Raúl; PERDOMO, Alfredo; DE LEÓN, Martín. 20 años de Puertas Abiertas. Devenires vitales en el Hospital Vilardebó. In: DE LEON, N. (Comp). Salud Mental en debate. Pasado, Presente y Futuro de las Políticas en Salud Mental. Montevideo: Psicolibros, 2013. p. 221-227. 
FOUCAULT, Michel. Microfísica del poder. Ciudad de México: Octaedro, 2003.

GOFFMAN, Erving. Estigma. La identidad deteriorada. Buenos Aires: Amorrortu, 1961.

PAGANO, Estefanía. Felisberto Hernández ¿Literatura Fantástica?. Montevideo, 2015. 14 p. Texto presentado en Seminario de Literatura Fantástica, Instituto de Profesores Artigas.

PERDOMO, Alfredo. Estar en el Vilardebó: una historia de experiencias emergentes. In: DE LEON, N. (Comp). Abrazos Experiencias y narrativas acerca de la locura y la salud mental. Montevideo: Ediciones Levy, 2013. p. $49-59$.

RODRÍGUEZ, Abelardo; SOBRINO, T. La rehabilitación psicosocial en el marco de una atención comunitaria integral al enfermo mental crónico. In: RODRÍGUEZ, A. (Comp.). Rehabilitación psicosocial de personas con trastornos mentales crónicos. Madrid: Ediciones Pirámide,1999. p. 330-369.

TOMMASINO, Humberto; RODRÍGUEZ, Nicolás. Tres tesis básicas sobre extensión y prácticas integrales en la Universidad de la República. In: Integralidad tensiones y perspectivas. Cuadernos de Extensión, n. 1. Montevideo: CSEAM, Universidad de la República, 2011. p. $19-42$.
ROMANO, Noemí. Bailanza, un neologismo, una propuesta posible. In: VEGH, I. (Comp.). Una cita con la psicosis. Rosario: Homo Sapiens Ediciones, 2007. p. 99-106.

TECHERA, Andrés; APUD, Ismael; BORGES, Cristina. La sociedad del olvido. Un ensayo sobre enfermedad mental y sus instituciones en Uruguay. Montevideo: CSIC, 2009.

UNIVERSIDAD DE LA REPÚBLICA. Rectorado. Hacia la Reforma Universitaria. La extensión en la renovación de la enseñanza. Espacios de Formación Integral. Montevideo, 2010. Disponível em: $<$ http://www.extension.udelar.edu.uy/wpcontent/uploads/2016/12/08 Hacia-la-reformauniversitaria -la-extensio $\% \overline{\mathrm{CC}} \% 81$ n-en-larenovacio $\% \overline{\mathrm{CC}} \% 81$ n-de-la-ensen $\% \mathrm{CC} \% 83$ anza.pdf $>$. Acesso em: 1 set. 2017.

VAN WEEGHEL, Jaap; KROON, Hans. Perfilando la investigación en rehabilitación psiquiátrica. Percepciones de los investigadores. Eur. J. Psychiat., Zaragoza, v. 18, n 2, p. 73-82, 2004. 